\title{
Review of: "Dynamic X-ray elastography using a pulsed photocathode source"
}

\author{
Christopher Vaughan ${ }^{1}$ \\ 1 University of Cape Town
}

Potential competing interests: The author(s) declared that no potential competing interests exist.

Although full-field digital mammography (FFDM) and more recently digital breast tomosynthesis (DBT) have been considered the gold standard for early detection of breast cancer, these X-ray systems often fail to detect malignant tumours, particularly in women with dense breast tissue [1]. This is because the lesion and the surrounding parenchymal tissue appear very similar in the X-ray image and the radiologist faces the challenge of "identifying a polar bear in a snowstorm" [2]. Since cancerous lesions are mechanically stiffer than adjacent healthy tissues, elastography has been shown to have considerable potential in detecting breast cancer [3].

In static elastography, a fixed pressure applied to the tissue of interest leads to localised strain (deformation) that can be mapped qualitatively using various imaging modalities [4]. However, because this technique does not measure the stress (load) within the tissue, it cannot provide a quantitative map of tissue stiffness [5]. In dynamic elastography, the application of time-varying pressure to the breast generates a shear wave within the tissue. The stress and strain produced by the shear wave may be continuously measured using an imaging modality such as ultrasound [6] although this modality lacks the spatial resolution of X-rays.

During the past decade, there have been a few studies published in which static elastography using X-ray imaging has been used $[7,8]$. The research team for the current study, led by Chika Kamezawa in Tsukuba, Japan and Rajiv Gupta in Boston, USA, is the first to apply dynamic X-ray elastography in which the tube was pulsed synchronously with an acoustic vibration device [9]. To test their system, the researchers manufactured a phantom from soft urethane resin that was doped with zirconium dioxide, while a hard inclusion $25 \mathrm{~mm}$ in diameter - to simulate a malignant tumour - was embedded within the centre of the phantom. The contrast-to-noise ratio (CNR) for the X-ray transmission image was 1.1, whereas the dynamic elastography technique for the same two rectangular regions of interest yielded a CNR of 4.5 , clearly demonstrating the feasibility of their system.

Furthermore, the storage modulus of elasticity of the hard inclusion as measured by dynamic X-ray elastography (12 $\mathrm{kPa}$ ) compared well with independent rheometer measurements (10 kPa), as did the 
values for the surrounding matrix (4.3 vs $3.4 \mathrm{kPa}$, respectively). These values are of the same order of magnitude as determined by dynamic ultrasound elastography [10] which reported shear elasticity values for breast cancer $(16.8 \mathrm{kPa})$ and normal breast tissue $(1.4 \mathrm{kPa})$. Again, this augurs well for the clinical feasibility of their approach.

The authors acknowledged that one limitation of their study was the large size $(25 \mathrm{~mm})$ of the simulated tumour and the need to study smaller lesions. They are confident their technique should not require higher radiation doses and that it could potentially be applied to existing FFDM and DBT systems. The next step will obviously be to conduct human clinical trials and we look forward to the outcome of that research.

\section{References}

[1] https://bit.ly/2wyzp7p

[2] https://bit.ly/3yWfAD4

[3] https://bit.ly/3]fmhVu

[4] https://bit.ly/32wCN2f

[5] https://bit.ly/33SkKV8

[6] https://bit.ly/3egLJeL

[7] https://bit.ly/3EmaHnU

[8] https://bit.ly/3Jo6Ydc

[9] https://go.nature.com/3Equ4Mm

[10] https://bit.ly/3Fr3Bzy 